CAHIERS DE

NARRATOLOGIE
Cahiers de Narratologie

Analyse et théorie narratives

$16 \mid 2009$

Images et récits

\title{
Récits d'Ellis Island (Georges Perec). Des récits contestés
}

Cécile de Bary

\section{CpenEdition}

Journals

Édition électronique

URL : http://journals.openedition.org/narratologie/942

DOI : $10.4000 /$ narratologie. 942

ISSN : 1765-307X

Éditeur

LIRCES

Référence électronique

Cécile de Bary, «Récits d'Ellis Island (Georges Perec). Des récits contestés », Cahiers de Narratologie [En ligne], 16 | 2009, mis en ligne le 26 mai 2009, consulté le 19 avril 2019. URL : http:// journals.openedition.org/narratologie/942; DOI : 10.4000/narratologie.942

Ce document a été généré automatiquement le 19 avril 2019

\section{(c) $($ i) $(9)$}

Cahiers de Narratologie - Analyse et théorie narratives est mis à disposition selon les termes de la licence Creative Commons Attribution - Pas d'Utilisation Commerciale - Pas de Modification 4.0 International. 


\title{
Récits d'Ellis Island (Georges Perec). Des récits contestés
}

\author{
Cécile de Bary
}

\section{NOTE DE L'ÉDITEUR}

Domaines de recherche de Cécile de Bary :

Littérature du $\mathrm{XX}^{\mathrm{e}}$ siècle

Littérature et cinéma. Littérature et arts plastiques.

Georges Perec, Oulipo et littératures à programmes : réception de ces œuvres.

Fiction et réception imaginaire du texte

Publications Cécile de Bary:

Direction du dossier « Jean-Patrick Manchette, un auteur hors série ? ", Temps noir, $\mathrm{n}^{\circ} 11$, 2008, p. 153-222.

Direction des Cahiers Georges Perec, n 9, Le Cinématographe, 2006.

«Reconnaissez-vous l'héritage de Queneau ? Perec et l'Oulipien que tout le monde connaît ", dans Connaissez-vous Queneau ? (actes du colloque international dirigé par Hela Ouardi et Marie-Noëlle Campana, Institut supérieur des sciences humaines, université Tunis-El Manar, 11-13 décembre 2003), Tunis/Dijon, Académie Beït al-Hikma/Presses universitaires de Dijon, 2007, p. 39-51.

«Le réel contraint », Poétique, n 144, novembre 2005, p. 481-489, 2005.

«L'intervention du vrai dans le pacte fictionnel : les invraisemblances de Raymond Queneau ", Revue des Sciences humaines, $n^{\circ} 280$, Le Vrai et le Vraisemblable, rhétorique et poétique, dir. Yves Le Bozec, octobre 2005, p. 117-130.

« Georges Polti, ou l'anticipation du théâtre potentiel », Poétique, n 138, avril 2004, p. 183-192.

Introduction

1 Récits d'Ellis Island ${ }^{1}$, film réalisé par Robert Bober et commenté par Georges Perec, porte sur un lieu emblématique de l'Histoire des États-Unis: jusqu'en 1924, les immigrants devaient passer par l'île d'Ellis Island avant d'entrer à New York. Dans la seconde partie 
de ce film (intitulée Mémoires), à laquelle je ne vais guère m'intéresser ici, Perec interviewe des témoins. La première partie ( Traces) met en perspective ces témoignages, voire les met à distance. Elle fait une large place au commentaire de Perec. Ce dernier, contrairement à ce que le titre pourrait laisser attendre, conteste le récit, ou plutôt les récits: Georges Perec, marqué par ce qu'il a appelé son "absence d'histoire ${ }^{2}$ ", se confronte aux légendes de l'immigration américaine et les met à distance. L'étude de la genèse de son commentaire permettra de montrer de quelle manière cette mise à distance opère et quelles en sont les conséquences, notamment sur le plan de l'énonciation.

\section{Perec et l'histoire}

2 Il convient tout d'abord de rappeler ce que recouvre cette "absence d'histoire » et son importance pour l'œuvre perecquienne. Dans un article de 1978, Perec fait de l'autobiographie l'un des "quatre axes» de son œuvre, avec le sociologique, le romanesque et le ludique (soit l'usage de contraintes oulipiennes'). Les chercheurs donnent cependant souvent à cet axe autobiographique une place centrale, notant en particulier combien le travail oulipien sur les formes chiffre certaines données exposées dans les textes personnels. W ou le Souvenir d'enfance, paru en 1975, devient de ce point de vue un ouvrage essentiel, alors même qu'il porte un discours autobiographique paradoxal, l'un des premiers chapitres énonçant : «Je n'ai pas de souvenirs d'enfance ${ }^{4}$.» L'absence d'histoire personnelle est la conséquence de l'intervention d' "une autre histoire, la Grande, l'Histoire avec sa grande hache ${ }^{5}$ ». Fils d'immigrés juifs polonais, né en 1936, Georges Perec perd son père, engagé volontaire, en 1940. Pour le protéger, sa mère se sépare de lui, avant d'être déportée en 1943. Perec a dès lors perdu jusqu'au souvenir de ses parents. Restent l'imaginaire et les histoires, qui lui apportent un secours malgré tout fragile ${ }^{6}$.

3 Cette «absence d'histoire » explique sans doute pourquoi Perec a été un autobiographe multiforme, qui a mis souvent en place des dispositifs complexes, comme Philippe Lejeune l'a montré. Cette écriture « oblique » est à mettre en relation avec une difficulté à dire « je ». Philippe Lejeune cite à ce propos un passage d'un projet inabouti, L'Âge :

Il faudrait dire Je. Il voudrait dire Je que ses mots déchirent les pages tracent leurs sillons noirs dans la vie même, mots brûlants d'une vertu qui ne s'éteindrait jamais

4 Dans la nouvelle des Lieux d'une fugue, qu'il adaptera au cinéma, Perec met en scène cette difficulté, puisque le récit y est écrit à la troisième personne, identifiée au «je " grâce à trois parenthèses qui complètent les trois dernières phrases ${ }^{8}$. Globalement, c'est une "lente émergence de la première personne ${ }^{9}$ " que permet un ensemble de projets autobiographiques très ambitieux, que Perec place au centre de son projet d'écrivain jusqu'en 1975, date de parution de $W$ ou le Souvenir d'enfance ${ }^{10}$. L'enjeu de ce dernier ouvrage est de décrire et d'inscrire: «le chemin que j'ai parcouru, le cheminement de mon histoire et l'histoire de mon cheminement ${ }^{11}$. "

Dans les films que Perec commente, l'énonciation peut également apparaître comme problématique. Ainsi, il se projette imaginairement dans les films amateurs que rassemble La vie filmée des Français. Vers la fin de l'épisode 1930-1934, période qui correspond presque à celle de ses premières années, il déclare : "J'ai découvert que j'aurais pu apparaître sur une de ces images ${ }^{12}$.» Or, Perec, qui lit lui-même son texte, ne se nomme pas dans ce commentaire. Ce n'est qu'au moment du générique de fin que les spectateurs peuvent éventuellement l'identifier. Le surgissement de ce «je » est donc surprenant, d'autant 
qu'il contrevient aux habitudes du genre documentaire. Qu'un réalisateur fasse un film autobiographique, cela se produit, mais il est plus rare qu'un commentateur de documentaire porte un discours propre ${ }^{13}$.

6 Les images sont ainsi le support d'histoires fictionnelles, ici comme souvent (les seuls souvenirs de ses parents que Perec ait pu reconstituer, ou réinventer, partent déjà de photographies). Et comme souvent encore, Perec insiste sur l'évanescence des images, images de films muets souvent figés dans le cliché, images qu'il présente comme mensongères. Il oscille entre une fascination et une critique d'images fragiles face aux fracas de la grande Histoire ${ }^{14}$.

7 Dans Récits d'Ellis Island, la mise en question du récit s'accompagne comme dans La vie filmée des Français de l'apparition de la première personne. Perec tient un discours personnel, évoquant sa judéité, sujet qu'il aborde explicitement pour la première fois. Son discours se fait donc autobiographique, dans un développement qui semble comme un point d'aboutissement, avant un mouvement conclusif ${ }^{15}$. Si ce film, diffusé en 1980 , et le livre sorti à la même époque ont été très étudiés ${ }^{16}$, la genèse du commentaire n'a guère été envisagée jusqu'à présent. Cette genèse est pourtant très particulière : elle conduit à une mise en crise des récits et à la mise en place de cette énonciation autobiographique. Comment Perec en est-il venu à parler de lui alors qu'il s'intéressait à l'immigration américaine? Comment «l'absence d'histoire " perecquienne est-elle intervenue à cette occasion? Ces questions ont des implications cinématographiques, puisqu'elles concernent également la collaboration avec Robert Bober.

II. Le film et son histoire

8 Le projet de Récits d'Ellis Island intervient après 1975, année de parution de W ou le Souvenir d'enfance et année du commentaire de La vie filmée des Français. Perec rencontre Robert Bober alors que celui-ci s'apprête à se rendre à Radom, en Pologne, sa ville paternelle. Dans le film qu'il tire de ce voyage, Réfugié provenant d'Allemagne, Apatride d'origine polonais $e^{17}$, celui-ci s'interroge sur le destin des juifs polonais. Après avoir vu ce film, Perec lui déclare qu'il aimerait qu'ils fassent un film «ensemble ». En septembre 1977, Bober lit dans un journal qu'Ellis Island est ouvert au public et propose à Perec d'y tourner un film.

9 Le réalisateur a apporté plusieurs témoignages sur cette collaboration ${ }^{18}$. On dispose également de nombreux avant-textes dans le fonds privé Georges Perec (qui rassemble les archives de l'écrivain), essentiellement dans le dossier $n^{\circ} 42^{19}$. Celui-ci comporte tout d'abord trois cahiers de petit format (A5).

10 Le premier cahier (cote 42,1), à couverture cartonnée vert foncé, présente un découpage de la première partie, de la main de Perec. Il ne s'agit pas toujours d'un découpage plan par plan, mais il est très détaillé, réservant une double page à chaque élément. La page de gauche indique le thème du fragment concerné et la page de droite est réservée au texte du commentaire. Les pages de droite sont le plus souvent vierges, le cahier n'ayant pas répondu à sa fonction jusqu'au bout.

11 Le deuxième cahier $(42,2)$, orange à spirales, a été tenu lors d'un premier voyage à New York à fin de repérages, dans la première quinzaine de juin 1978. Perec, dans Repérages, la troisième partie du livre, décrit des pratiques d'écriture qui correspondent au contenu de ce cahier :

Nous étions partis de Paris avec quelques dizaines d'adresses. Très vite nos carnets furent bourrés d'autres noms, d'autres adresses, d'autres numéros de téléphone : 
des gens à voir, des institutions à contacter, des renseignements à demander, des

formalités à accomplir ${ }^{20}$. indication de découpage du film, annotée au crayon de papier dans la marge de gauche. On remarque encore :

- la présentation des témoins de la deuxième partie, soit presque exactement le texte du commentaire de cette partie, beaucoup plus réduit que celui de la première partie $(42,23,23$ à 27) ;

- des manuscrits et des tapuscrits correspondant au générique, par exemple 42, 22,1; ou 42, $23,7,1-2 d$;

- des manuscrits et des tapuscrits correspondant à d'autres textes liés au projet et au livre : le tapuscrit de «Ellis Island, description d'un projet », publié dans Le Catalogue pour des juifs de maintenant en septembre $1979^{25}$; le tapuscrit de passages de "L'île des larmes ", première partie du livre qui n'est pas dite dans le film (42,23,12 à 16, feuillets manifestement en désordre), etc. correspondant aux Récits d'Ellis Island dans un entretien qu'il a accordé à Frank Venaille : 
sa parole retranscrite devient un élément exogénétique ${ }^{26}$. Ce dossier est donc riche, quoiqu'incomplet. Ainsi, un journal correspondant à la traversée que Perec fit en bateau vers les États-Unis pour rejoindre le tournage (publié autrefois par la revue TEM) se trouve dans le dossier $106^{27}$. Un album, dont je reparlerai, a d'autre part été utilisé lors du tournage. Il est en la possession de Robert Bober, qui a cependant autorisé de nombreuses reproductions, notamment dans Perec images et dans la réédition du livre Récits d'Ellis Island ${ }^{28}$. Le dossier 42 , centré sur l'écriture de Perec, est composé d'éléments successifs souvent repris ultérieurement et donc reclassés.

III. Des histoires des immigrants à l'histoire d'un film

19 L'articulation entre le destin de Perec et celui des immigrés se joue autour de la notion d'histoire. Un manuscrit collé ultérieurement dans l'album est une liste d'histoires, les histoires fondatrices d'Ellis Island ${ }^{29}$. Avant l' Histoire du bohémien fabriquant des cigares et l' Histoire de Christophe Colomb, les deux premières sont l' Histoire de Robert Bober et celle de Perec. Comme je l'ai dit, ces histoires devaient certainement être considérées comme structurantes par les protagonistes du projet, puisque Robert Bober a déclaré que les quinze feuillets initiaux concernaient des histoires. Le titre y renvoie encore.

Les histoires des immigrants fascinent manifestement Perec, comme toutes les histoires empreintes d'imaginaire, mais qu'il le veuille ou non, il est du côté du manque et de l'absence: quand il interroge les immigrants, dans la deuxième partie de Récits d'Ellis Island, il oublie systématiquement comment on dit « souvenir » en anglais. Dans un autre ordre d'idées, Robert Bober a souvent insisté sur la symbolique du choix de certaines photos de l'album déjà mentionné qui sont montrées à l'image: la photo du salon de coiffure abandonné de la mère de Perec faisant face à celle de la famille réunie du réalisateur, autour de son grand-père ${ }^{30}$.

21 Avec l'immigration américaine, Perec est face à un mythe: les destins des immigrants s'articulent imaginairement à l'Histoire, grâce à leur dimension légendaire. L'une des histoires recensées par le manuscrit collé dans l'album est d'ailleurs celle «du rêve américain $^{31}$ ». Sur un autre manuscrit, Perec note :

Ce que nous ressassons

ces anecdotes

quatre fois par jour répétees

par des vieux scouts $\left(42,3,58 \mathrm{v}^{\circ}\right)$

Il refuse ce ressassement, ce qui est apparent lorsqu'il désigne la répétition « une fois de plus» des paroles du guide, qui délivre des histoires que ses auditeurs «connaissent depuis toujours ${ }^{32}$ ». Andrée Chauvin et Mongi Madini ont ainsi montré comment Perec ne prend guère en charge le narratif et le met à distance. On observe une même distance à l'égard des images, ce qui rejoint le procédé de Robert Bober qui, en montrant des photographies tirées à grande échelle sur les lieux où elles ont été prises, fait ressortir l'absence des immigrants et fait réfléchir à la valeur de témoignage des images ${ }^{33}$. La première partie est ainsi présentée dans la Déclaration d'intentions :

Dans une première partie, qui pourrait durer approximativement une heure, il s'agira pour nous, à travers la sensibilité personnelle que nous avons de cette époque et de cette histoire, de nous situer par rapport à ce lieu: nous nous efforcerons de restituer, à l'aide de textes et de documents, aussi abondants que précis, et par une visite d'Ellis Island, devenue aujourd'hui Musée de l'immigration, ce que fut la vie quotidienne dans ce centre de transit [...]. $(42,23,11,2 \mathrm{~d}$.) photographiques mis en scène in situ par Robert Bober. Le discours factuel est encore plus 
absent du commentaire : Andrée Chauvin et Mongi Madini ont montré qu'il était délégué pour l'essentiel au ranger qui effectue la visite guidée que suit dans l'ensemble la première partie $^{34}$.

Ces critiques ont encore remarqué que le documentaire est plus descriptif que narratif ${ }^{35}$. Il fait une grande place à une interrogation très perecquienne, qui rejoint une fois encore son interrogation autobiographique, celle de l'infra-ordinaire: il s'agit de parvenir à retrouver la quotidienneté, ce qui ne laisse normalement pas de traces et que la grande Histoire oublie ${ }^{36}$. Cette interrogation est dubitative, d'où une mise en crise du narratif qui correspond à l'absence d'histoire et de tradition du côté de Perec :

comment décrire?

comment raconter?

comment regarder ? [...]

Comment saisir ce qui n'est pas montré, ce qui n'a pas été photographié, archivé, restauré, mis en scène?

Comment retrouver ce qui était plat, banal, quotidien, ce qui était ordinaire, ce qui se passait tous les jours? [...]

cela ne veut rien dire de vouloir faire parler ces images, de les forcer à dire ce qu'elles ne sauraient dire.

au début, on ne peut qu'essayer de nommer les choses, une à une, platement [...]

c'est ce que l'on voit aujourd'hui

et l'on sait seulement que ce n'était

pas ainsi au début du

siècle ${ }^{37}$.

Le commentaire porte autant sur la démarche de Bober et Perec que sur les immigrants. Il ne s'agit plus seulement de faire un film sur l'histoire des immigrants mais d'interroger le film lui-même, d'évoquer « le chemin ${ }^{38}$ » parcouru, de retracer l'histoire du film.

IV. De l'histoire du film à l'histoire personnelle

D'après les classements du premier tapuscrit «thématique", Perec a dès le début envisagé de commencer son texte par un fragment intitulé Les deux questions, fragment qui correspond au tout début du film (juste après le générique, avant même l'intertitre annonçant la première partie, Traces), début où l'on voit Perec de dos, en train de feuilleter l'album:

À Paris, quand nous disions que nous allions faire un film sur Ellis Island, presque tout le monde nous demandait de quoi il s'agissait. À New York, presque tout le monde nous demandait pourquoi. Non pas pourquoi un film à propos d'Ellis Island, mais pourquoi nous. En quoi cela nous concernait-il, nous, Robert Bober et Georges Perec ?

Il serait sans doute un peu artificiel de dire que nous avons réalisé ce film à seule fin de comprendre pourquoi nous avions le désir ou le besoin de le faire. Il faudra bien, pourtant, que les images qui vont suivre répondent à ces deux questions et décrivent, non seulement ce lieu unique, mais le chemin qui nous y a conduits ${ }^{39}$.

Si Perec évoque son histoire (ou son absence d'histoire), c'est donc sans doute pour répondre à la deuxième de ces questions. Le passage concerné est d'ailleurs introduit par une série de phrases interrogatives qui détaillent celle-ci ${ }^{40}$. La dimension personnelle est aussi inscrite dès le début de la mise en œuvre du projet, à travers le journal. Ce journal, notamment lors des repérages, est ensuite une trace d'un parcours qui s'inscrit dans le commentaire. Un exemple en est donné par la séquence d'arrivée sur l'île :

c'est le mercredi 31 mai 1978 que nous avons pour la première fois, Robert Bober et moi, visité Ellis Island. En même temps que nous, il y avait un couple d'une cinquantaine d'années, dont la mère de la femme, une Roumaine qui était passée 
par Ellis Island, était morte quelque temps auparavant, et une toute jeune femme qui tenait dans ses bras un bébé âgé de quelques jours à peine.

ce n'est jamais, je crois, par hasard, que l'on va aujourd'hui visiter Ellis Island. Ceux qui y sont passés n'ont guère eu envie d'y revenir. Leurs enfants ou leurs petitsenfants y retournent pour eux, viennent y chercher une trace : ce qui fut pour les uns un lieu d'épreuves et d'incertitudes est devenu pour les autres un lieu de leur mémoire, un des lieux autour desquels s'articule la relation qui les unit à leur histoire ${ }^{41}$.

La remarque qui débute le deuxième mouvement s'applique autant à Bober et Perec qu'aux autres visiteurs de l'île : c'est la suite de la « deuxième question ». En tout cas, le début réécrit un passage du journal, daté du 31 mai $\left(42,2,6 \mathrm{r}^{\circ}\right)$. Le passage et sa genèse sont caractéristiques d'une volonté de rendre compte autant du parcours des immigrants que de celui des concepteurs du film, qui se marque également dans les deux dates 1978-1980 - inscrites entre parenthèses au générique ${ }^{42}$.

À cette volonté rétrospective correspond la composition de l'album, qui comporte des textes manuscrits, des prospectus, de nombreuses photographies, photographies d'époque mais aussi photographies de tournage, et encore photographies personnelles de Perec et de Bober. L'idée de l'album est venue de Perec ${ }^{43}$. D'après son agenda de 1980 (97, $9,2,0$ ), les séquences concernant l'album ont été tournées alors que le montage était presque fini et après l'enregistrement du commentaire. Si sa présence était prévue plus tôt, puisqu'il figure dans le découpage du cahier vert, il s'agit bien d'une trace construite a posteriori ${ }^{44}$.

30 L'insistance sur l'implication de Robert Bober, et surtout de Georges Perec, a de plus conduit le commentateur à produire un discours curieux, portant en particulier sur sa relation avec la judéité. Outre que la présence d'un tel discours ne correspond pas aux habitudes du genre documentaire, celle-ci a conduit à une difficulté d'interprétation, conduisant certains interprètes à faire d'Ellis Island une métaphore de la Shoah.

V. L'implication du commentateur

Cette interrogation pourrait d'une certaine manière avoir été inscrite dès le début du projet, puisque Perec cherchait aussi, à travers lui, à se confronter à Robert Bober et à son rapport à la judéité, différent du sien. Travailler avec lui, c'était envisager une question personnelle. Le réalisateur a également indiqué que les premiers temps de préparation du film, avant le premier voyage à New York, ont surtout consisté en des rencontres fréquentes, où les deux cherchaient à mieux se connaître l'un l'autre ${ }^{45}$. Le passage autobiographique qui nous préoccupe est suivi d'un passage où il parle de Bober. Selon le témoignage de ce dernier, Perec lui a demandé d'écrire un texte sur ce que c'était pour lui d'être juif, puis l'a réécrit pour l'intégrer à son commentaire.

Si la première personne était d'emblée prévue dans le projet, elle devait y avoir une moindre importance, comme le montre le tapuscrit «thématique ». Si l'on se reporte à la Déclaration d'intentions, on y observe un discours personnel, qui intervient à la fin, et qui sera ensuite pleinement réintégré au projet de la première partie :

Nous avons l'un et l'autre de la famille en Amérique et c'est en partie par hasard que nos [sic] grands-parents ou nos parents, dans leur quête de la terre promise, se sont arrêtés et fixés en France.

C'est en ce sens que nous nous impliquons dans ce projet et qu'il s'inscrit pour nous dans la démarche commune que, par des moyens différents, nous poursuivons à la recherche de notre histoire. $(42,23,11,2 \mathrm{~d}$.) 

mémoire potentielle, d'une autobiographie probable. » (Je cite Récits d'Ellis Island ${ }^{46}$.) On a donc l'impression d'une identification avec les immigrants. Perec n'a-t-il pas voyagé comme eux par bateau? Aux photos des immigrants, qu'ils montrent volontiers dans la deuxième partie, correspondent les photos personnelles de Perec et Bober, présentes dans l'album. Dans son agenda, Perec envisageait un cahier, mais c'est finalement bien un album qui a été filmé : s'il y appose son écriture, réécrivant des fragments qu'il a déjà prononcés, les images, surtout des photos, dominent. Elles ne sont pas reconnaissables par le spectateur, sauf, s'il est averti, pour la photo du salon de coiffure de la mère de Perec tel qu'il apparait dans les années soixante-dix ${ }^{47}$. Mais le spectateur perçoit qu'il s'agit de traces correspondant à celles dont chacun dispose sur sa propre histoire, celles d'un album de famille.

Aller aux États-Unis, pour Perec, c'est se confronter de manière très concrète à la possibilité d'un autre destin. Perec mentionne parfois dans ses avant-textes certains de ses cousins qui sont passés par Ellis Island. C'est surtout Simone qu'il fréquente. À la fin du cahier marron, il note dans une page intitulée « Simone » $\left(42,3,63 v^{\circ}\right)$ que l'histoire de celle-ci « est en de nombreux points identique à la mienne ». Seule différence, « Simone et ses parents avaient quitté la France à la fin 1941.» Le début du passage rappelle les « ritournelles » et les « litanies » dont Régine Robin a parlé à propos du projet de L'Arbre 48 : «La mère de Simone est la sœur du mari de la sœur de mon père », etc. Dans plusieurs manuscrits, Perec semble retrouver ce projet, centré autour d'une histoire de sa famille. Il en reprend au moins les modalités d'inscription. Il dessine ainsi deux arbres généalogiques, l'un qui concerne essentiellement la famille Bienenfeld, l'autre qui relie David Bienenfeld à Simone et qui comporte une esquisse du texte précédent ${ }^{49}$. " concerne bcp _ " (42, 3,16, « dimanche 13 » mai 1979). Le dernier chapitre est consacré à la manière dont les immigrants considèrent le destin auquel ils ont échappé en immigrant aux États-Unis et s'appuie sur de nombreux témoins dont les proches ont péri dans la Shoah ${ }^{50}$.

On peut donc comprendre que Perec se soit investi personnellement dans son commentaire. Cet investissement suscite cependant une difficulté interprétative, qui explique que Récits d'Ellis Island soit souvent lu en référence à l'Histoire " avec sa grande hache ", le centre de transit devenant une métaphore des camps. C'est une interprétation très fréquente, mais qui dérange de nombreux proches, à commencer par Robert Bober. Perec lui-même s'y oppose dans un entretien qu'il donne au moment de la sortie du film :

Dans Traces, on ne peut s'empêcher de penser, en voyant la façon inhumaine dont sont traités les émigrés dans ce "parc à bestiaux» suicidaire (marquages, changements de nom, séparation des malades, etc.), à l'arrivée dans les camps nazis...

G. P. - Il faut rappeler d'abord que les gens qui avaient mis deux ans et toutes leurs économies à préparer leur voyage aux USA arrivaient tout de même à Ellis Island pleins d'espoir dans une vie nouvelle. L'arrivée est terrible car il y a cette incertitude : il faut passer l'épreuve de «l'île des larmes »... Mais les déportés qui arrivaient dans les camps n'étaient pas pleins d'espoir. Ce n'est donc pas comparable ${ }^{51}$.

Il n'est certes pas question de donner le dernier mot à Perec parce qu'il est l'auteur du commentaire. De fait, ce hiatus entre une interprétation dramatique d'Ellis Island et une réalité somme toute positive court au long de nombreux manuscrits qui oscillent entre les 
deux visions. Dans un feuillet manuscrit glissé dans le carnet marron, Perec insiste sur l'espoir :

Ellis Island n'était pas un lieu tragique

Il ne s'y passait rien d'horrible ou de terrible

L'Ile des larmes était surtout île d'espoir

Seuil d'une vie nouvelle

Partir en Amérique $(42,3,65+\mathrm{d})$

Dans la version publiée, le texte d'abord assez neutre devient dramatique (faisant écho à la fin du chapitre VI de $W$ ou le Souvenir d'enfance, qui évoque Hitler, puis précise que la situation pour Perec et les siens « allait bientôt devenir vitale, c'est-à-dire, le plus souvent mortelle $\left.{ }^{52} »\right)$ :

il ne s'agit pas de s'apitoyer mais de comprendre quatre émigrants sur cinq n'ont passé sur Ellis

Island que quelques heures

ce n'était, tout compte fait, qu'une formalité anodine,

le temps de transformer l'émigrant en immigrant,

celui qui était parti en celui qui était arrivé,

mais pour chacun de ceux qui défilaient

devant les docteurs et les officiers d'état civil,

ce qui était en jeu était vital :

ils avaient renoncé à leur passé et à leur histoire,

ils avaient tout abandonné pour tenter de venir vivre

ici une vie qu'on ne leur avait pas donné le droit de

vivre dans leur pays natal

et ils étaient désormais en face de l'inexorable ${ }^{53}$

39 J'ai retranscrit dans les Annexes 1 et 2 les avant-textes de ce passage. (Voir aussi la reproduction de l'un d'entre eux, le ms 42, 23,36, dans l'Annexe 2.) Le tapuscrit «thématique » $(42,23,9,2)$ commence par une notation pessimiste, celle de la périphrase "l'̂̂le des larmes ", que l'on retrouvera développée au début du commentaire ${ }^{54}$. Dans le paragraphe suivant, cependant, une auto-injonction refuse l'émotion au profit de la compréhension. Le manuscrit 42, 23,36 (reproduit en Annexe 2) reprend cette dernière idée et l'illustre, détaillant "ce qui s'est passé » pour "ceux qui sont passés", euphémisant de surcroît les formalités administratives en «formalité anodine». Suit alors un paragraphe qui débute par un «mais », orienté vers une conclusion inverse (qui correspond au $4^{\mathrm{e}}$ paragraphe du texte final tel que je l'ai cité ci-dessus). Plus encore, sur le manuscrit, on remarque un mouvement d'ajout ${ }^{55}$, avec un autre instrument d'écriture, un crayon de papier, à partir de l'adjectif « inexorable» (qui semble prendre le relais de «vital»). Bien que refuser de céder à l'émotion, Perec revient comme fatalement aux notations de douleur ; il est même tenté de les développer.

À l'inverse, déléguant toute une partie de l'information à un guide officiel, tenu à une certaine neutralité consensuelle, il évacue les soubassements idéologiques de la sélection. Dans la première partie que Perec a rédigé pour le livre, «L'île des larmes ", il évoque les quotas et leur mise en place à partir de $1914^{56}$. Or, pourquoi imposer des quotas, si ce n'est en fonction de préjugés racistes? On pourrait citer à ce propos Island of Hope, Island of Tears, que Perec a lu attentivement ${ }^{57}$.

Dans le même passage, Perec évoque rapidement l'existence de tests d'intelligence, à partir de 1917. Ceux-ci sont détaillés par exemple dans un autre film consacré à Ellis Island, fictionnel celui-là, Golden Door, d'Emmanuele Crialese ${ }^{58}$. Ce que Crialese fait énoncer, au prix de différentes invraisemblances historiques et scénariques, c'est que 
l'emploi de tests d'intelligence reposait sur des motivations eugéniques. Perec n'insiste donc pas sur le tri des hommes et encore moins sur ses motivations, telles qu'elles apparaissent au début du XX $\mathrm{XX}^{\mathrm{e}}$ siècle ${ }^{59}$. Comme nous l'avons vu, c'est la notion d'histoire qui sert de pivot entre le destin personnel de Perec et celui des immigrants.

Myriam Soussan a montré la résonance d'un lieu en ruine pour quelqu'un dont le destin a été brisé ${ }^{60}$. Cette valeur métaphorique est très apparente dans le passage qui m'a particulièrement retenue, dans lequel Perec évoque précisément ce destin, de manière $a$ priori incongrue. Un des plans, presque abstrait, correspond particulièrement bien au texte qui évoque « quelque chose d'informe, à la limite du dicible ${ }^{61}$ ».

Robert Bober a évoqué une interrelation très fine entre eux deux au moment du montage, expliquant qu'il avait réalisé certains plans fixes pour laisser à Perec le temps de les décrire, ou encore qu'il avait doublé un plan pour laisser assez de place pour le passage qui le concernait lui-même dans son rapport à la judéité ${ }^{62}$. Le montage tel qu'il apparaît dans le cahier vert a surtout été bouleversé pour un passage, celui qui concerne la judéité de Perec. J'ai tenté de faire ressortir ces changements dans un tableau qui compare les annotations marginales du tapuscrit 42, 23,8 avec le montage définitif (voir Annexe 3). J'ai composé en gras les plans qui ont été placés plus tôt et en italiques ceux qui apparaissent plus tard. Même si l'on ne peut être absolument certain de l'identification de tous les plans (une « salle avec piliers » étant difficile à distinguer d'une autre), il est certain que la séquence a été globalement rallongée, à l'aide d'ajouts de plans (soulignés). Le film laisse ainsi place au silence, ce qui peut être rapproché de la mise en page de l'ouvrage, avec ses vers libres qui se détachent sur le blanc de la page. Par ailleurs, on observe que le «plan abstrait » a été déplacé, sans doute du fait de la conscience de sa possible portée métaphorique, dans sa nouvelle correspondance avec le texte. Enfin, le plan long " couloir puis pano sur un autre couloir » correspond à un passage essentiel, caractérisé par une cohésion du sens. L'absence de coupe a pour effet de ne pas disperser l'attention pour celui-ci. L'unité du plan correspond à une unité du texte. Dans ce passage, le montage est donc au service du commentaire, ce qui n'est pas toujours le cas. Ainsi, dans le troisième cahier, Perec, qui a effectué un découpage minuté, note à côté de "Photo salle des bagages » : « Texte à trouver » $\left(42,3,50 \mathrm{r}^{\circ}\right)$.

C'est donc bien d'une co-autorité qu'il s'agit, ce que montre le générique de début du film ${ }^{63}$. Perec, présent au tournage comme au montage, compose les sous-titres, accompagne le film jusqu'à sa diffusion, écrivant des textes pour des revues. C'est alors une énonciation plurielle que le film met en place. Dès le début du commentaire, c'est d'un «nous » qu'il est question, «nous » répété sept fois dans le premier (petit) paragraphe avant que soit clairement énoncée la double identité de Robert Bober et Georges Perec: ce "nous " permet d'éviter le flottement ressenti à la vision de La vie filmée des Français. Il fait de Perec le porte-parole de Bober: dans le passage qui concerne la judéité de l'un («je ») puis de l'autre («il »), leurs noms sont redits ; ce passage consacré à chacun succède à un passage en « nous » (« pourquoi racontons-nous ces histoires ? » etc.) Quant à l'album, qui apparait lors de moments stratégiques, il permet sans doute de soutenir cette énonciation personnelle à deux voix, en faisant du film dans son ensemble le lieu d'un parcours rétrospectif, critique et subjectif.

Conclusion

45 L'étude génétique de Récits d'Ellis Island permet donc de saisir les tensions qui sont à l'œuvre au cœur de son projet, avec notamment une contradiction entre fascination et refus des histoires. De même, la visée documentaire croise la visée autobiographique, 
alors que la première partie est destinée à introduire un autre film, où ce sont les immigrants qui ont la parole : Robert Bober et Georges Perec parlaient pour la première partie de «notre film» et pour la deuxième de «leur film ${ }^{64}$ ». Si Perec peut tenir un discours personnel dans cette première partie, c'est que Bober lui laisse l'occasion de le faire. Il est ainsi un véritable co-auteur. Leur collaboration est si forte que le livre, qui contient surtout le texte du commentaire, est signé par les deux. Perec réarticule dans Récits d'Ellis Island des obsessions personnelles, ainsi de l'absence d'histoire et des fracas de la grande Histoire: comme dans $W$ ou le Souvenir d'enfance, il y retrace «le cheminement de [s]on histoire et l'histoire de [s]on cheminement. » Robert Bober place au centre du film une interrogation sur la photographie présente dès Réfugié provenant d'Allemagne, Apatride d'origine polonaise, interrogation qui se poursuivra dans l'hommage qu'il fera bien plus tard à son ami, En remontant la rue Vilin ${ }^{65}$.

\section{NOTES}

1. Récits d'Ellis Island, histoires d'errance et d'espoir, réalisation de Robert Bober, scénario et commentaire de Georges Perec, production INA, 1979, couleur, 2 parties, $1 \mathrm{~h} 56 \mathrm{~min}$. Première diffusion sur TF 1 les 25 et 26 novembre 1980. Les auteurs ont publié en même temps un livre : Robert Bober et Georges Perec, Récits d'Ellis Island, histoires d'errance et d'espoir, Paris, Le Sorbier/INA, 1980. (Rééd. P.O.L/INA, 1994.)

2. Georges Perec, W ou le Souvenir d'enfance, Paris, 1975, rééd. Gallimard, coll. «L'imaginaire », 1993, p. 17.

3. Georges Perec, « Notes sur ce que je cherche », Le Figaro, 8 décembre 1978, rééd. in Penser/Classer, Paris, Hachette, coll. « Textes du XXe siècle », 1985, p. 9-12.

4. Georges Perec, W ou le Souvenir d'enfance, op. cit., p. 17.

5. Ibid.

6. Au chapitre XXXI de W ou le Souvenir d'enfance, Perec évoque certains épisodes de ses lectures d'enfant qui lui « ont presque servi d'histoire » : « les livres racontaient des histoires ; on pouvait suivre ». Il remarque toutefois ensuite que ses livres préférés étaient incomplets, « en impliquaient d'autres, absents, et introuvables ». Ibid., p. 195.

7. Philippe Lejeune, La Mémoire et l'Oblique, Georges Perec autobiographe, Paris, P.O.L, 1991, p. 24-25.

8. Par exemple, pour l'antépénultième : « Lorsque vingt ans plus tard, il entreprit de se souvenir (lorsque vingt ans plus tard, j'entrepris de me souvenir) » (« Les lieux d'une fugue », nouvelle datée de 1965 et publiée en 1975, rééd. in Je suis né, Paris, Seuil, coll. « La librairie du xxe siècle », 1990, p. 30). Dans le film, les phrases sont directement à la première personne, la nouvelle étant toujours globalement à la troisième personne. Les Lieux d'une fugue (« Caméra-je »), réalisation de Georges Perec, voix de Marcel Cuvelier, production INA, 1978, couleur, $41 \mathrm{~min}$.

9. Expression de Philippe Lejeune, à propos du projet de L'Âge, loc. cit.

10. Sur cette deuxième période de l'écriture autobiographique de Perec et sur le programme qu'il élabore à partir de 1968, voir Philippe Lejeune, ibid., p. 36-48.

11. W ou le Souvenir d'enfance, op. cit., p. 18. 
12. Il s'agit d'une série qui s'appuie sur des films amateurs réalisés avec la caméra Pathé, de façon à évoquer différentes époques historiques. Perec a commenté l'épisode suivant : 1930-1934 (« La vie filmée des Français », 2 e partie), réalisation de Michel Pamart et Claude Ventura, commentaire de Georges Perec, production INA, 1975, noir et blanc, $52 \mathrm{~min}$. Le texte du commentaire ( $«$ La vie filmée ») a été édité dans les Cahiers Georges Perec, $\mathrm{n}^{\circ} 9$, 2006, p. 73-82.

13. D'après François Jost : « Le documentaire s'oppose au film de fiction, en cela qu'il suppose généralement une coïncidence entre le locuteur premier, le grand imagier qui organise le film, et ce locuteur second qu'est ce commentateur souvent anonyme, qui explique, sémantise, complète, ce que nous voyons. » (" ... Mon tout est une fiction », p. 47, dans Cinémas et Réalités, Saint-Étienne, université de Saint- Étienne, Centre interdisciplinaire d'étude et de recherche sur l'expression contemporaine (CIEREC), coll. « Travaux », 1984.)

14. Sur ce commentaire, voir mon article « Une mémoire fabuleuse ? De l'Histoire à l'histoire ", actes du colloque international Georges Perec et l'Histoire (université de Copenhague, 30 avril- $1^{\text {er }}$ mai 1998), Études romanes, $n^{\circ} 46,2000$, p. 9-20. Voir, plus généralement, ma thèse : Image, Imagination, Imaginaire dans l'œuvre de Georges Perec, sous la direction de Philippe Hamon, université Paris III-Sorbonne nouvelle, 2002.

15. La fin du commentaire est consacrée aux « suites » de l'île : la fermeture des frontières américaines au cours de la première moitié $\mathrm{du} \mathrm{XX}^{\mathrm{e}}$ siècle, le devenir des immigrants une fois entrés aux États-Unis.

16. Voir les « Références » du n 9 des Cahiers Georges Perec, op. cit, p. 293-311. En particulier, Mongi Madini et Andrée Chauvin ont réalisé une étude très complète des relations du livre et du film dans « La remontée des images », Le Cabinet d'amateur, $\mathrm{n}^{\circ} 6$, 1997, p. 39-81.

17. Réfugié provenant d'Allemagne, Apatride d'origine polonaise, réalisation de Robert Bober, production INA, 1975, noir et blanc, $1 \mathrm{~h} 30$ min.

18. Voir notamment l'entretien avec Robert Bober paru dans le $n^{\circ} 9$ des Cahiers Georges Perec, « Le regard et l'absence », op. cit., p. 245-254.

19. Sur le fonds privé Georges Perec, déposé à la bibliothèque de l'Arsenal, voir Hans Hartje, «Les archives Perec », Cahiers de textologie, nº 4, Configurations d'archive, 1993, p. 109-134. Un inventaire complet du fonds a depuis été effectué par Éla Bienenfeld, Bianca Lamblin et Paulette Perec. Je remercie Éla Bienenfeld, de la confiance qu'elle m'a accordée en m'autorisant la consultation d'une partie de ce fonds, ainsi que la citation et la reproduction de certains avant-textes de Récits d'Ellis Island.

20. Il s'agit de la troisième partie dans la première édition de 1980, op. cit. Dans la réédition de 1994, op. cit, une troisième partie constituée de photographies, intitulée « Album », a été ajoutée. « Repérages » est donc la quatrième partie, extrait cité p. 94.

21. Le cahier a été repris ultérieurement, comme le montre le feuillet $42,2,39 \mathrm{v}^{\circ}$ utilisé à l'envers pour une entrée de journal datée du $1^{\mathrm{er}}$ mai 1979. Le feuillet $42,2,39 \mathrm{r}^{\circ}$, utilisé à l'envers, comporte par exemple un avant-texte du Cabinet d'amateur. On trouve des brouillons de tautogrammes sur les feuillets 42,2,27 et 28.w Par ailleurs, une feuille qui comporte des indications de minutage (et donc contemporaine de l'écriture du commentaire au moment du montage) est glissée dans le carnet : 42,2,43+d.

22. $20,9 \times 17,4 \mathrm{~cm}$. Le format indiqué par la couverture du carnet est donné en pouces : $8^{1}$ $/ 4 \times 6^{7} / 8$. Carnet de la marque « National ».

23. David M. Brownstone, Irene M. Franck and Douglass Brownstone, Island of Hope, Island of Tears, New-York (USA), Wade Publishers Inc, 1979. (Rééd. Barnes \& Noble, 2000.) 
24. Op. cit., p. 247.

25. «Ellis Island, description d'un projet », Recherches, $n^{\circ} 38$, Catalogue pour des Juifs de maintenant, septembre 1979, p. 51-54. (Rééd. dans Je suis né, op. cit., 1990, p. 95-103.) Ts 42,23,6,1 à 5, avec un feuillet classé ailleurs, le feuillet 42,19 (dernier feuillet du tapuscrit, numéroté 6 par Perec lui-même).

26. 42,4. Feuillet daté par Perec du 4 mars 1979. «Perec ou le contraire de l'oubli », entretien avec Frank Venaille, Monsieur Bloom, n³, mars 1979, p. 72-75. Rééd. in Je suis né, op. cit. Le passage concerné se trouve p. 85-86.

27. 106,1,1,1 à 13. Reproduit dans Texte en main, $\mathrm{n}^{\circ}$ 12, Perec, Polaroïds, printemps 1997, p. 35-44.

28. Jacques Neefs et Hans Hartje, Georges Perec, images, Paris, Seuil, 1993.

29. Manuscrit reproduit dans Georges Perec images, op. cit., p. 172.

30. «Le regard et l'absence », loc. cit., p. 250.

31. Loc. cit.

32. Récits d'Ellis Island, op. cit., p. 50.

33. A. Chauvin et M. Madini, « La remontée des images », loc. cit., p. 55.

34. Ibid., p. 49-52.

35. Ibid., p. 68-74.

36. Voir " Approches de quoi ? ", Cause commune, n 5, février 1973, rééd. in L'Infraordinaire, Paris, Seuil, coll. « La librairie du XX siècle », 1989, p. 9-13.

37. Récits d'Ellis Island, histoires d'errance et d'espoir, rééd. P.O.L/INA, op. cit., p. 37-45. 38. Le texte du commentaire est intitulé dans le livre « Description d'un chemin ». On retrouve donc l'expression du chapitre II de W ou le Souvenir d'enfance, op. cit., p. 18.

39. Je reproduis ici le texte du manuscrit figurant sur l'album, tel qu'il est reproduit dans l'édition de 1994, op. cit., p. 5. Il correspond exactement au texte que Perec prononce dans le film. Le texte - composé - paru en 1980 est légèrement différent (alors qu'il est en général fidèle au commentaire tel qu'il est lu). Le nom «Bober » a tout d'abord été oublié et la toute fin est différente, d'abord parce que le texte - situé dans une position paratextuelle, après la page de titre et avant la page d'épigraphe et de dédicace introduit un livre et non plus un film, ensuite pour des raisons qui semblent stylistiques : «Il faudra bien, pourtant, que les images et les textes qui vont suivre rendent compte, non seulement de ce que fut Ellis Island, mais du chemin qui nous y a conduits. » (Op. cit., p. 7.) Le tapuscrit intitulé « Les deux questions » $(42,23,9,1)$ a d'abord été classé par Perec à l'aide de la lettre $\mathrm{A}$, puis du chiffre 1 . Il est très semblable au texte définitif, malgré d'importantes modifications stylistiques, sur lesquelles il n'est pas de mon propos de m'attarder. Par ailleurs, un plan figurant sur le ms 42,11,1 commence par « 1 les 2 questions ».

40. "pourquoi racontons-nous ces histoires ?/ que sommes-nous venus chercher ici ?/ que sommes-nous venus demander?» (Récits d'Ellis Island, rééd. 1994, op. cit., p. 55.)

41. Ibid., p. 36.

42. Dans le cahier vert, ces dates ne sont pas inscrites, ni dans le tapuscrit du générique. Elles apparaissent dans un seul avant-texte, le manuscrit 42,23,7,2d.

43. D'après Robert Bober, « Faire un film ensemble », loc. cit., p. 12. Voir aussi « Le regard et l'absence ", loc. cit., p. 249.

44. À la date du 19 février 1980, Perec écrit dans son agenda : «Enr. Texte Ellis Isl $1^{\text {ere }}$ " $(97,9,2,30)$. Et pour le 25 : « Enreg. Texte Ellis» $\left(97,9,2,33 r^{\circ}\right)$. Pour le 27 février au matin : « Tournage cahier » $\left(97,9,2,34 \mathrm{r}^{\circ}\right)$. En date du 16/2, Perec envisage l'achat du cahier pour 
$12 \mathrm{~h}$ : « Bober achat cahier » $\left(97,9,2,27 \mathrm{v}^{\circ}\right)$. Il biffe d'un autre stylo cette indication, stylo qu'il garde pour entourer le 14 , notant en face : « B. chez moi » $\left(97,9,2,28 \mathrm{v}^{\circ}\right)$.

Pour ce qui est du cahier vert, dès le feuillet $42,1,1 \mathrm{v}^{\circ}$, Perec note « Le cahier ». En face $\left(2 \mathrm{r}^{\circ}\right)$, on trouve le même texte que celui cité plus haut.

45. Voir Robert Bober, « Faire un film ensemble », TEM, n 12, op. cit., p. 11.

46. Loc. cit., p. 55. Citation située au début du passage autobiographique. On peut considérer l'entretien avec Frank Venaille que j'ai mentionné à la note 26 comme un avant-texte de ce passage.

47. Cette photo où se lit l'enseigne « Coiffure Dames », figure sur la couverture originale de $W$ ou le Souvenir d'enfance, op. cit.

48. Régine Robin, «Un projet autobiographique inédit de Georges Perec : L’Arbre », Le Cabinet d'amateur, $\mathrm{n}^{\circ} 1,1998$, p. 10.

49. Le premier est situé dans le cahier orange $\left(42,2,4 \mathrm{v}^{\circ}\right)$. Le deuxième figure au dos d'une facture d'hôtel qui correspond aux quatre premiers jours de mai $1979\left(42,7 \mathrm{v}^{\circ}\right)$. Sur les arbres généalogiques de L'Arbre, voir Régine Robin, ibid.

50. Island of Hope, Island of Tears, op. cit., p. 281-284 en particulier.

51. « Georges Perec et Robert Bober : "Ellis Island, c'est le temps où les États-Unis incarnaient la Terre promise" ", propos recueillis par Jean Liberman, novembre 1980, rééd. in Entretiens et Conférences, édition critique établie par Dominique Bertelli et Mireille Ribière, [Nantes], Joseph K., 2003, volume II, p. 141. Voir aussi la note 9 de l'ouvrage, qui porte sur ce passage.

52. W ou le Souvenir d'enfance, op. cit., p. 36.

53. Récits d'Ellis Island, op. cit., p. 52.

54. Ibid., p. 28. Le tapuscrit est d'abord classé à l'aide de la lettre B, puis de 2 : il a longtemps été prévu de le faire figurer au début du commentaire.

55. Ajout que commente en marge une indication de régie peut-être antérieure, puisqu'écrit avec le même type de feutre que le début du texte : « developper ».

56. Op. cit., p. 11 et 15-16.

57. P. 16, les auteurs d'Island of Hope, Island of Tears citent par exemple Zane Grey (The Call of the Canyon, 1922), qui évoque un " suicide racial et la horde des immigrants étrangers » (" race suicide and the incoming horde of foreigners »).

58. Golden door (Nuovomundo), réalisation et scénario d'Emmanuele Crialese, avec Vincenzo Amato, Charlotte Gainsbourg et Aurora Quattrocchi, 2006, Rai Cinemafiction, 1 h $58 \mathrm{~min}$.

59. Sur ces sujets, voir aussi les publications et documents correspondant à l'exposition Augustus Frederick Sherman : Ellis Island portraits 1905-1920 produite par la fondation Aperture (New York) et accueillie à la Cité nationale de l'histoire de l'immigration fin 2007-début 2008. Voir en particulier le site de la Cité : http://www.histoireimmigration.fr/index.php?lg=fr\&nav=596\&flash=0, dernière consultation en janvier 2008. 60. Myriam Soussan, «La mémoire vivante des lieux : Perec et Bober », Le Cabinet d'amateur, http://www.cabinetperec.org/articles/soussan/artsoussan.html, dernière consultation en mai 2007.

61. Récits d'Ellis Island, op. cit., p. 56. Voir l'analyse d'Andrée Chauvin et Mongi Madini, loc. cit., p. 66-67.

62. « Le regard et l'absence ", loc. cit., p. 248.

63. Il y est question d' " un film de Robert Bober et Georges Perec ».

64. Robert Bober, « Le regard et l'absence », loc. cit., p. 248. 
65. En remontant la rue Vilin, réalisation de Robert Bober, texte de Robert Bober, avec des textes de Georges Perec, coproduction VF Films Production, La Sept, INA et Centre audiovisuel de Paris, 1992, couleur/noir et blanc, 49 min.

\section{RÉSUMÉS}

Le film que Robert Bober et Georges Perec ont consacré à l'îlot situé face à New York par lequel sont passés la plupart des immigrants de la fin du XIXe siècle et du début du XXe siècle est constitué de deux parties, la deuxième étant consacrée aux témoignages d'anciens immigrants. La première partie, objet de cet article, met les témoignages de ceux-ci en perspective, puisqu'elle est construite autour de plusieurs interrogations, par exemple sur la possibilité de restituer leur histoire, ou sur la relation que les auteurs du film entretiennent avec celle-ci. Je souhaiterais étudier le commentaire de ce film à partir de son dossier génétique qui figure dans les archives Perec (fonds privé en dépôt à la BNF). On voit grâce à lui comment le projet s'appuie d'abord sur des histoires multiples pour de plus en plus les mettre à distance: le descriptif domine dès lors et le commentaire, qui énonce aussi un discours autobiographique à deux voix. Perec y explicite pour la première fois ce que représente pour lui la judéité : refusant de «ressasser» les légendes de l'immigration américaine, le trop-plein des anecdotes, il se confronte à son histoire brisée, marquée par l'absence.

\section{INDEX}

Mots-clés : fiction, Perec Georges, judéité, critique génétique, Bober Robert, documentaire, commentaire cinématographique, légende, mythe, États-Unis, histoire, récit, autobiographie, immigration, Ellis Island

Index chronologique : XXe siècle

\section{AUTEUR}

\section{CÉCILE DE BARY}

Maître de Conférences, Université de Nice-Sophia Antipolis, Chercheure au CTEL (Centre transdisciplinaire d'épistémologie de la littérature - EA 1758). 